\title{
Aquaporin-4-antibody-positive Neuromyelitis Optica Spectrum Disorder in a Patient with Charcot-Marie-Tooth Disease Type 1A
}

\author{
Yuichi Hamada, Kazusa Takahashi, Takamichi Kanbayashi, Yuki Hatanaka, \\ Shunsuke Kobayashi and Masahiro Sonoo
}

\begin{abstract}
:
Charcot-Marie-Tooth disease type 1A (CMT1A) is a hereditary peripheral neuropathy, and its involvement in the central nervous system (CNS) is very rare. We herein report a 51-year-old woman with CMT1A who suffered from recurrent optic neuritis and myelopathy. Under the diagnosis of anti-aquaporin-4 (anti-AQP4) antibody positive neuromyelitis optica spectrum disorder (NMOSD), we treated her successfully with corticosteroids. This is the first report of CMT1A complicated with anti-AQP4-positive NMOSD. Although the coexistence of the two disorders may simply be a coincidence, we speculated that immune cross-reaction between overexpressed peripheral myelin protein 22 and CNS myelin may have caused concomitant CMT1A and NMOSD.
\end{abstract}

Key words: Charcot-Marie-Tooth disease type 1A, neuromyelitis optica spectrum disorder, optic neuritis, anti-aquaporin-4 antibody

(Intern Med 60: 1611-1614, 2021)

(DOI: 10.2169/internalmedicine.6153-20)

\section{Introduction}

Charcot-Marie-Tooth disease (CMT) is a group of inherited motor sensory neuropathies. Demyelination in the central nervous system (CNS) is a rare complication of CMT, except for in its X-linked subtype (CMTX), in which CNS involvement has occasionally been reported (1).

We herein report a patient with CMT type 1A (CMT1A) who suffered from recurrent optic neuritis and myelopathy. We discuss the immune mechanisms underlying the concomitant involvement of the peripheral nervous system and the CNS.

\section{Case Report}

A 51-year-old Japanese woman was admitted to our hospital because of left ophthalmic pain and progressive blindness during the previous 9 days. One year before this presentation, she had experienced repeated numbness and pain in the lower extremities, which spontaneously remitted after a couple of weeks. One month before the admission, she had experienced blurred vision and discomfort of the right eye, which improved spontaneously after several days. She had experienced gait disturbance since childhood and had developed atrophy and paresthesia of the distal lower extremities during adolescence. She had a family history of peripheral neuropathy with the autosomal dominant form of inheritance (Fig. 1). The disease onset was in adolescence in all affected members, except for in a sister of the patient, whose onset was at elementary school age.

On admission, her left eye was blind with a dilated pupil and an absent light reflex. A fundoscopic examination revealed redness of the left optic nerve papilla. The right eye had visual acuity of 0.80 (Snellen fraction of 20/25) with a normal light reflex and a normal fundoscopic appearance. The critical flicker fusion threshold (CFF) was unmeasurable in the left eye and $34 \mathrm{~Hz}$ in the right eye. Distal extremities exhibited wasting and weakness, especially the lower extremities. There was sensory loss of all modalities in the dis- 


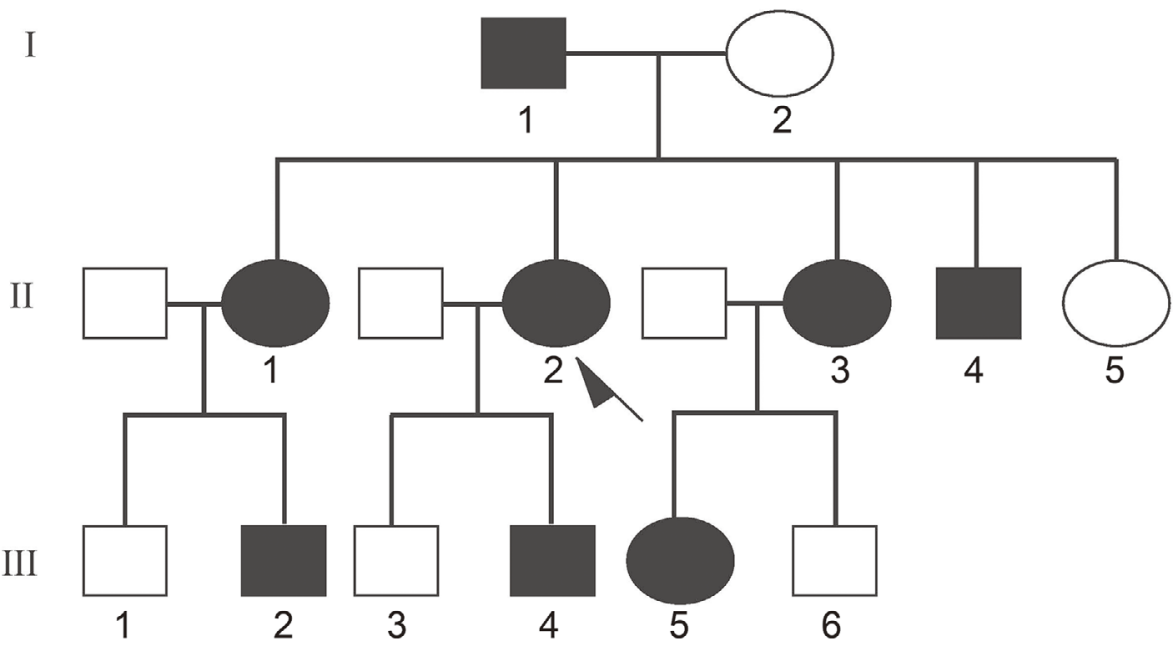

Figure 1. Pedigree tree of the family. Filled symbols indicate individuals with peripheral neuropathy and pes cavus. The father of the patient (I-1) had had difficulty running since he was a teenager. The older sister of the patient (II-1) had suffered from neuropathy since elementary school. Two siblings (II-3, 4), two nephews (III-2, 4), and one niece (III-5) of the patient had developed neuropathy in their teenage years. No family members had disorders involving the central nervous system, including optic neuritis. The arrow indicates the proband. Squares and circles indicate men and women, respectively.

tal lower limbs. All deep tendon reflexes were absent, and pathological reflexes were not elicited. Pes cavus and hammer toe were noticed. She could walk without support but exhibited high steppage due to foot drop. Magnetic resonance imaging (MRI) revealed T2 elongation in the left optic nerve, and multiple high-intensity T2-weighted lesions were present in the cerebral deep white matter. Long spinal cord lesions were also observed on MRI (Fig. 2). Gadolinium-enhanced images were not obtained.

Nerve conduction studies indicated systemic demyelinating neuropathy (Table 1). Visual evoked potentials (VEPs) were not detectable on left eye stimulation, and the latency was mildly prolonged in the right eye. Blood test results were unremarkable except for serum positivity for aquaporin-4 antibody (anti-AQP4). Serum myelin oligodendrocyte glycoprotein antibody (anti-MOG) was negative. Autoantibodies, including antinuclear antibody, SS-A, SS-B, and $\mathrm{Sm}$, were negative. Thyroid hormones were normal. Cerebrospinal fluid (CSF) showed a normal protein level (18 $\mathrm{mg} / \mathrm{dL}$ ) without pleocytosis or oligoclonal bands (OCB). The IgG index was mildly elevated (0.84; normal limit $0.65)$. A genetic analysis revealed duplication of the peripheral myelin protein 22 (PMP22) gene.

We diagnosed the patient with anti-AQP4-positive neuromyelitis optica spectrum disorder (NMOSD) plus underlying CMT1A. We treated her with three courses of methylprednisolone pulse therapy and seven plasma exchanges. The visual acuity of the left eye recovered to 0.03 (Snellen fraction of 20/630), and the CFF became measurable $(20 \mathrm{~Hz})$.

During maintenance therapy with $30 \mathrm{mg}$ oral prednisolone, the patient experienced worsening of paresthesia of the extremities, hand tremor, and gastric ulcer. These symptoms subsided after reducing oral prednisolone and adding azathioprine.

\section{Discussion}

We encountered a case of recurrent optic neuritis and myelitis with long-standing sensorimotor polyneuropathy. The clinical picture of the chronic neuropathy in this patient was typical of CMT1A, and the diagnosis was confirmed genetically. Patients with CMT1A typically do not present with visual symptoms. However, subclinical prolongation of VEP is reported in 10-16\% of CMT1A patients, which may be associated with asymptomatic optic neuropathy (2). Apart from the above-mentioned degenerative optic neuropathy, acute inflammatory processes in the CNS are an extremely rare complication of CMT1A.

To our knowledge, only eight cases of acute CNS inflammatory lesions have been reported in association with CMT1 A (3-8). Of these, four cases of CMT1A with optic neuritis are summarized in Table 2. In one case, antibodies to the proteolipid protein (PLP) and MOG were positive, but antiAPQ4 was not reported in these previous cases. A CSF analysis was positive for OCB in three cases, and proteincell dissociation was present in two cases. Neither OCB nor protein-cell dissociation was present in our patient. The IgG index was often elevated including our patient, suggesting inflammation in the CNS. These reports raise the possibility that the abnormal expression of PMP22 in peripheral nerve and demyelination of the CNS are causally related. One hypothesis is that PMP22 shares homology with other CNS myelin proteins, such as the PLP, and that the PMP22 overexpression in CMT1A induces an autoimmune response in the CNS $(1,6)$. PLP has been studied extensively as a relevant primary antigen in multiple sclerosis (MS), and the T- 

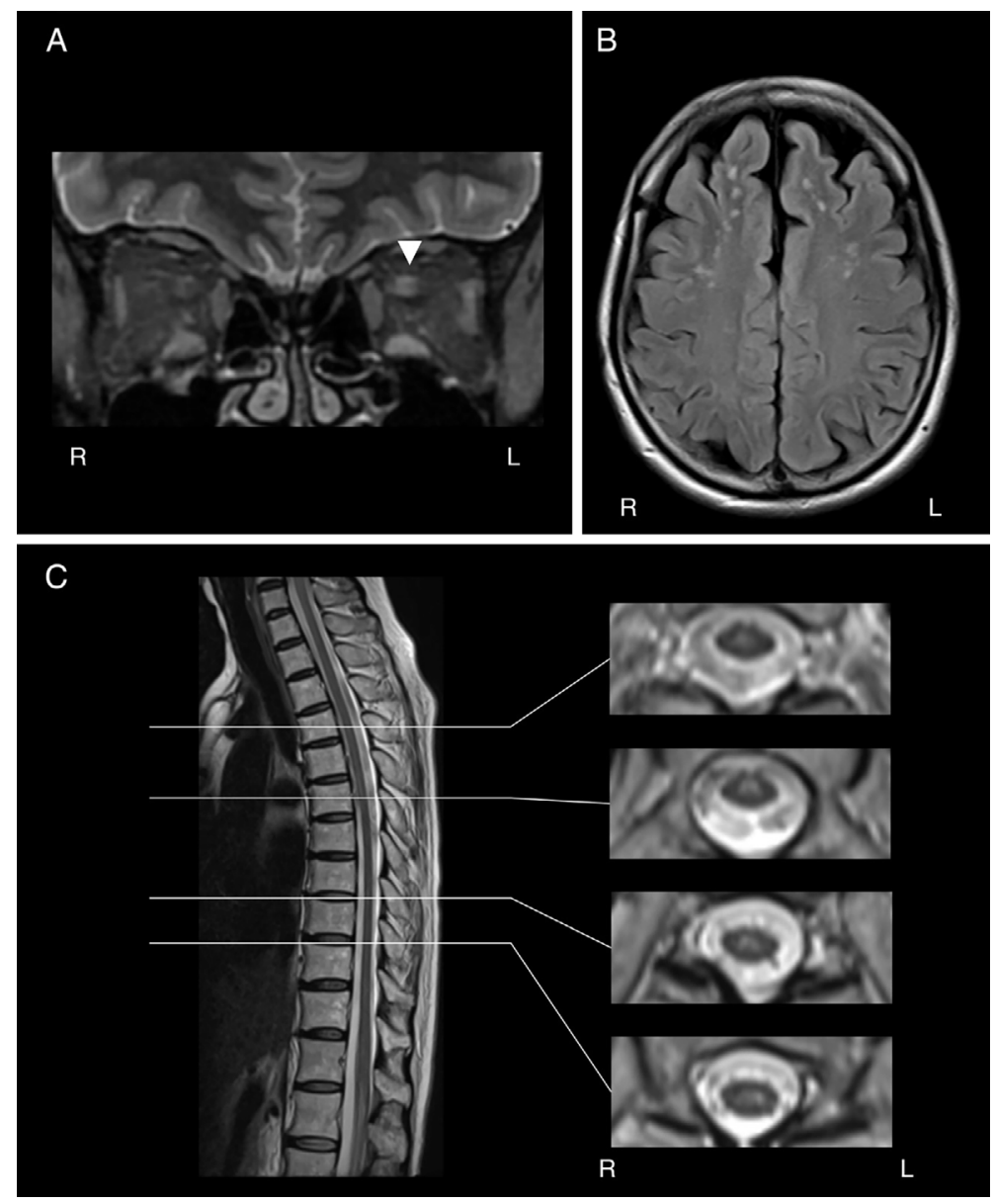

Figure 2. MRI of the brain and spinal cord. (A) A coronal T2-weighted image shows high-intensity signals in the left optic nerve (arrowhead; TE=21.6 ms/TR=7,300 ms). (B) An axial FLAIR image of the brain shows multiple high-intensity spots in the white matter (TE=105.3 ms/TR=8,000 ms). (C) A midsagittal image of the spinal cord with axial sections at the levels indicated by the white lines (Th4, Th6, Th9, and Th10 from top to bottom). Longitudinal hyperintense lesions are evident in the spinal cord $(\mathrm{TE}=103.0 \mathrm{~ms} / \mathrm{TR}=3,000 \mathrm{~ms})$.

Table 1. Electrophysiological Findings.

\begin{tabular}{llccc}
\hline & Nerve & Amp $(\mathrm{mV}, \mu \mathrm{V})$ & DL $(\mathrm{ms})$ & $\mathrm{CV}(\mathrm{m} / \mathrm{s})$ \\
\hline \multirow{2}{*}{ MCSs } & Median & $7.8^{*}$ & $11.4^{*}$ & $24^{*}$ \\
& Ulnar & $4.2^{*}$ & $8.4^{*}$ & $24^{*}$ \\
& Tibial & $0.05^{*}$ & $13.2^{*}$ & $18^{*}$ \\
SCSs & Median & $1.0^{*}$ & $7.04^{*}$ & $18^{*}$ \\
& Ulnar & $0.6^{*}$ & $5.66^{*}$ & $20^{*}$ \\
& Sural & $0.5^{*}$ & $8.52^{*}$ & $18^{*}$ \\
\hline \multirow{3}{*}{ VEPs } & Side & \multicolumn{3}{c}{ P100 latency (ms) } \\
\cline { 2 - 5 } & Right & \multicolumn{3}{c}{$121.2^{*}$} \\
& Left & \multicolumn{3}{c}{ Not evoked* }
\end{tabular}

Nerve conduction studies were performed on the right median, ulnar, tibial, and sural nerves. Asterisks indicate significant results with reference to the standard values in our institute. Unit for amplitude is mv for MCSs and $\mu \mathrm{V}$ for SCSs. Amp: amplitude, CV: conduction velocity, DL: distal latency, MCSs: motor nerve conduction studies, SCSs: sensory nerve conduction studies, VEPs: visual evoked potentials

cell response to PLP epitope induces experimental autoimmune encephalomyelitis $(9,10)$. Wakerley et al. (6) ob- served increased immunity against PLP and MOG epitopes in their patient, supporting the immune cross-reactivity hypothesis. There are clinical conditions that suggest a pathophysiological link between peripheral and central myelin; (i) Pelizeus-Merzbacher disease is a leukodystrophy caused by the overexpression of PLP protein gene and is known to be associated with CMT-like neuropathy (11), (ii) hereditary neuropathy with liability to pressure palsy is caused by a deletion or mutation in PMP22 and known to manifest CNS white matter lesions (12).

NMOSD was previously considered a variant of MS but is now recognized as a distinct clinical entity. In MS, T cells are thought to target PLP expressed in oligodendrocytes. In contrast, NMOSD is primarily mediated by B-cell autoimmunity to AQP4, with AQP4-IgG antibodies binding to AQP 4 expressed on astrocytes and activating the complement pathway (13). However, T-cell immunity in NMOSD is receiving increasing attention. For example, Matsuya et al. reported that PLP induced a significant T-cell response in NMOSD, suggesting that immunity to myelin proteins plays a pathological role (14). Michalski et al. reported that small 
Table 2. Previously Reported Cases of Optic Neuritis with CMT1A.

\begin{tabular}{|c|c|c|c|c|c|c|c|c|}
\hline Reference & $\begin{array}{c}\text { Age } \\
\text { (years)/ } \\
\text { Sex }\end{array}$ & Diagnosis & $\mathrm{OCB}$ & $\begin{array}{l}\operatorname{IgG} \\
\text { index }\end{array}$ & $\begin{array}{l}\text { CSF } \\
\text { cell }\end{array}$ & $\begin{array}{c}\mathrm{CSF} \\
\text { protein } \\
(\mathrm{mg} / \mathrm{dL})\end{array}$ & Demyelination on MRI & VEPs \\
\hline 3 & $38 / \mathrm{F}$ & MS & + & 0.89 & ND & ND & ND & Prolonged \\
\hline 3 & $30 / \mathrm{F}$ & MS & + & 0.98 & ND & ND & $\begin{array}{l}\text { Cerebral white matter and } \\
\text { spinal cord }\end{array}$ & Prolonged \\
\hline 6 & $57 / \mathrm{M}$ & $\begin{array}{l}\text { Optic neuritis } \\
\text { (anti-MOG) }\end{array}$ & + & ND & Normal & 1,290 & Only optic nerve & Prolonged \\
\hline 1 & 31/M & MS & - & 0.65 & Normal & 52 & $\begin{array}{l}\text { Cerebral white matter and } \\
\text { spinal cord }\end{array}$ & ND \\
\hline Present case & $51 / \mathrm{F}$ & $\begin{array}{c}\text { NMOSD } \\
\text { (anti-AQP4) }\end{array}$ & - & 0.84 & Normal & 18 & $\begin{array}{l}\text { Optic nerve, cerebral white } \\
\text { matter and spinal cord }\end{array}$ & Prolonged \\
\hline
\end{tabular}

F: female, M: male, anti-MOG: anti-myelin oligodendrocyte glycoprotein antibody, MS: multiple sclerosis, ND: not described, OCB: oligoclonal band, CSF: cerebrospinal fluid, VEPs: visual evoked potentials, anti-AQP4: anti-aquaporin-4 antibody, NMOSD: neuromyelitis optica spectrum disorder

amounts of PLP were expressed in astrocytes in mice (15). To our knowledge, the present report is the first of CMT1A complicated with anti-AQP4-positive NMOSD. Thus, the coexistence may simply be coincidental. However, an immune cross-reaction remains a potential explanation, and further research is needed.

Our patient experienced exacerbation of paresthesia in the lower extremities after corticosteroid maintenance therapy was initiated, which we attributed to peripheral neuropathy based on the sensory distribution. It is unclear why corticosteroid therapy elicited sensory neuropathy in this CMT1A patient. We speculated that methylprednisolone upregulated the already-overexpressed PMP22 via its effect as a progesterone receptor agonist and consequently worsened the CMT1A symptoms. Progesterone is known to regulate the expression of PMP22; for example, a progesterone antagonist reduced PMP22 overexpression in a mouse model of CMT1A and improved the severity of the symptoms (16).

There is accumulating evidence that CMT1A is a risk factor of MS. Together with the findings of a previous report of anti-MOG-positive optic neuritis (6), the present results raise the possibility that CMT1A is also a risk factor for B-cellmediated CNS demyelination. Further clinical and basic immunological studies are needed to elucidate the mechanisms underlying the immune cross-reaction between the peripheral nervous system and the CNS.

The authors state that they have no Conflict of Interest (COI).

\section{References}

1. Koros C, Evangelopoulos ME, Kilidireas C, et al. Central nervous system demyelination in a Charcot-Marie-Tooth type $1 \mathrm{~A}$ patient Case Rep Neurol Med 2013: 243652, 2013.

2. Bird TD, Griep E. Pattern reversal visual evoked potentials. Studies in Charcot-Marie-Tooth hereditary neuropathy. Arch Neurol 38: 739-741, 1981 .
3. Frasson E, Polo A, Di Summa A, et al. Multiple sclerosis associated with duplicated CMT1A: a report of two cases. J Neurol Neurosurg Psychiatry 63: 413-414, 1997.

4. Almsaddi M, Bertorini TE, Seltzer WK. Demyelinating neuropathy in a patient with multiple sclerosis and genotypical HMSN-1. Neuromuscul Disord 8: 87-89, 1998.

5. Panas M, Karadima G, Kalfakis N, et al. Charcot-Marie-Tooth disease type 1A with central nervous system involvement in two generations. J Neurol 251: 484-485, 2004.

6. Wakerley BR, Harman FE, Altmann DM, et al. Charcot-MarieTooth disease associated with recurrent optic neuritis. J Clin Neurosci 18: 1422-1423, 2011.

7. Doğan Y, Gül S, Ceylan AC, et al. A special association between Charcot-Marie-Tooth type $1 \mathrm{~A}$ disease and relapsing remitting multiple sclerosis. Mult Scler Relat Disord 35: 83-85, 2019.

8. García-Estévez DA, Cid-Rodríguez C, Ozaita-Arteche G. CharcotMarie-Tooth Disease Type $1 \mathrm{~A}$ and Inflammatory-Demyelinating Lesions in the Central Nervous System. Int J Neurol Neurother 6: 080, 2019.

9. Davies S, Nicholson T, Laura M, et al. Spread of T lymphocyte immune responses to myelin epitopes with duration of multiple sclerosis. J Neuropathol Exp Neurol 64: 371-377, 2005.

10. Greer JM, Pender MP. Myelin proteolipid protein: an effective autoantigen and target of autoimmunity in multiple sclerosis. J Autoimmun 31: 281-287, 2008.

11. Anderson TJ, Klugmann M, Thomson CE, et al. Distinct phenotypes associated with increasing dosage of the PLP gene: implication ns for CMT1A due to PMP22 gene duplication. Ann N Y Acad Sci 883: 234-246, 1999.

12. Tackenberg B, Möller JC, Rindock H, et al. CNS involvement in hereditary neuropathy with pressure palsies (HNPP). Neurology 67: 2250-2252, 2006.

13. Häusser-Kinzel $S$, Weber MS. The role of $B$ cells and antibodies in multiple sclerosis, neuromyelitis optica, and related disorders. Front Immunol 10: 201, 2019.

14. Matsuya N, Komori M, Nomura K, et al. Increased T-cell immunity against aquaporin-4 and proteolipid protein in neuromyelitis optica. Int Immunol 23: 565-573, 2011.

15. Michalski J-P, Anderson C, Beauvais A, et al. The proteolipid protein promoter drives expression outside of the oligodendrocyte lineage during embryonic and early postnatal development. PLoS One 6: e19772, 2011.

16. Sereda MW, Meyer zu, Hörste G, Suter U, et al. Therapeutic administration of progesterone antagonist in a model of CharcotMarie-Tooth disease (CMT-1A). Nat Med 9: 1533-1537, 2003.

The Internal Medicine is an Open Access journal distributed under the Creative Commons Attribution-NonCommercial-NoDerivatives 4.0 International License. To view the details of this license, please visit (https://creativecommons.org/licenses/ by-nc-nd/4.0/).

(C) 2021 The Japanese Society of Internal Medicine Intern Med 60: 1611-1614, 2021 